\title{
Synaptonemal complexes of univalent B chromosomes in the grasshoppers Euthystira brachyptera and Myrmeleotettix maculatus
}

\author{
Hugh L. Fletcher* and \\ Godfrey M. Hewitt $\dagger$
}

\author{
* Genetics Department (Biology), \\ The Queen's University of Belfast, Belfast BT7 1NN, \\ Northern Ireland. \\ $\dagger$ School of Biological Sciences, \\ The University of East Anglia, Norwich NR4 7TJ, \\ U.K.
}

\begin{abstract}
B chromosomes of two species of grasshoppers, Myrmeleotettix maculatus and Euthystira brachyptera, have been studied. The synaptonemal complexes of univalent $B$ chromosomes in male meiosis are described. The results support the view that they are isochromosomes, and there is no evidence of differences in behaviour of the $B$ between the two species.
\end{abstract}

\section{INTRODUCTION}

B chromosomes have been described in many species where they are supernumerary to the regular complement. Their structure, effects on recombination, phenotype and evolution are the subjects of considerable research (Jones and Rees, 1982) but in several aspects they remain enigmatic. Stable B chromosomes are often iso-chromosomes or iso-chromosome derivatives, being metacentric, or submetacentric, with their arms identical or similar. In Myrmeleotettix maculatus the arms of a single submetacentric B chromosome will pair with each other during meiosis and form a chiasma to give a ring at metaphase I (John and Hewitt, 1965). When two or three B's are present they can frequently form bivalents or trivalents; as well as univalents. This suggests that having homologous arms held together by the centromere does not greatly facilitate foldback pairing. In meiotic prophase these Bs are heterochromatic and consequently their pairing may be advanced in time or different in some other way from the autosomes. B chromosomes in most organisms are usually heterochromatic (Jones and Rees, 1982) and a B chromosome very similar in appearance and meiotic behaviour to that in M. maculatus has been reported in Euthystira brachyptera (Fletcher and Hewitt, 1980).

Virtually nothing is known about the ultrastructure of pairing and chiasma formation in such B's and so the synaptonemal complexes of univalent B chromosomes in male meiosis in both species are described below. Both species have a normal male complement of 3 long metacentrics, 5 telocentrics, and an $\mathrm{X}$.

\section{MATERIALS AND METHODS}

Light microscopy: Part of the follicular testis was fixed in 3 parts ethanol, 1 part acetic acid. Squash preparations were made in lacto-propionic orcein. Electron microscopy: The remainder of the testis was fixed in 4 per cent gluteraldehyde, stained in 1 per cent phosphotungstic acid in absolute ethanol, embedded in Spurr's resin, and serial sectioned. $3 \mathrm{D}$ reconstructions were made of the cells from photographs of the serial sections. See Fletcher (1977) for details of method.

\section{RESULTS}

The relative mitotic lengths of the chromosomes are shown in table 1 . The synaptonemal complex (SC) lengths from a late pachytene stage of 1B $M$. maculatus are shown, with a complete early pachytene and a partial zygotene SC karyotype of a OB E. brachyptera, and the ranked complete SC's from two zygotene cells from a $1 \mathrm{~B}$ E. brachyptera individual. One of these latter cells was from the 
Table 1 Lengths of mitotic chromosomes and meiotic synaptonemal complexes from Myrmeleotettix maculatus and Euthystira brachyptera. The $M$. maculatus mitotic data is abstracted from two published $0 \mathrm{~B}$ and two published $1 \mathrm{~B}$ photographs of C-mitoses (John and Hewitt, 1965b; John and Hewitt, 1966). The mitotic lengths for E. brachyptera were measured in seven second meiotic metaphase cells, six containing B chromosomes, and two very clear 0B embryonic C-mitoses. At MII the short chromosomes seem relatively shorter than in mitosis. Errors range from $+/-5$ per cent for the long chromosomes to $+/-12$ per cent for the shorter ones. All per cent lengths are per cent of the normal autosomal karyotype, that is neglecting the B and X chromosomes.

\begin{tabular}{|c|c|c|c|c|c|c|c|c|c|c|}
\hline \multicolumn{11}{|c|}{ Chromosome number } \\
\hline Species/cell & L1 & $\mathrm{L} 2$ & L3 & M4 & M5 & M6 & S7 & S8 & B & \\
\hline \multicolumn{11}{|c|}{ Myrmeleotettix maculatus } \\
\hline $\begin{array}{l}\text { Mitotic \% All } \\
\text { Meiotic } \mu \mathrm{m} \mathrm{SC}\end{array}$ & $27 \cdot 1$ & 24 & $19 \cdot 7$ & $9 \cdot 2$ & $7 \cdot 0$ & $5 \cdot 3$ & $4 \cdot 3$ & $3 \cdot 36$ & $12 \cdot 3$ & $(6 \cdot 1)^{*}$ \\
\hline $1 \mathrm{~B} / 1$ & 83 & 81 & 66 & 29 & 24 & 14 & 12 & 11 & 10 & $(3 \cdot 1 \%)$ \\
\hline \multicolumn{11}{|c|}{ Euthystira brachyptera } \\
\hline $\begin{array}{l}\text { Mitotic \% All } \\
\text { Meiotic } \mu \mathrm{m} \mathrm{SC}\end{array}$ & $24 \cdot 6$ & $22 \cdot 3$ & $20 \cdot 6$ & $10 \cdot 2$ & $8 \cdot 0$ & $6 \cdot 2$ & $4 \cdot 8$ & $3 \cdot 2$ & $8 \cdot 1$ & $(4 \cdot 0)^{*}$ \\
\hline $0 \mathrm{~B} / 1$ & 116 & 107 & 100 & 57 & 40 & 30 & 25 & 20 & & \\
\hline$(\%)$ & $23 \cdot 4$ & $21 \cdot 6$ & $20 \cdot 2$ & $11 \cdot 5$ & $8 \cdot 1$ & $6 \cdot 1$ & $5 \cdot 1$ & $4 \cdot 0$ & & \\
\hline $0 \mathrm{~B} / 2$ & & & & 49 & 37 & 29 & 23 & 19 & & \\
\hline
\end{tabular}

* The length of the B is also shown halved for comparison with the foldback SC.

earliest cyst containing SC's. The ranked proportional lengths of SC's is in good agreement with the relative lengths of mitotic chromosomes.

The SC associated with the B univalents is the shortest SC in the nucleus and can be identified by three criteria. (i) in E. brachyptera the shortest $\mathrm{SC}$ in the $1 \mathrm{~B}$ cells has no counterpart in the $\mathrm{OB}$ cells. (ii) The precocious condensation of the $B$ is visible (fig. 1(a), (b) and (d)) in all cells, particularly in the late pachytene cell of M. maculatus. (iii) No more than one end of the SC of the B chromosome is attached to the nuclear envelope (fig. 1(a) and (c)). This is presumably the telomeric end (See fig. 2(c)). In the E. brachyptera cells which were at an early stage of meiosis (zygotene) and therefore with less advanced condensation, the centromere or kinetochore was visible near the unattached end of the SC where the chromatin was adpressed to the envelope. At this end, it became impossible to follow the SC, and in one E. brachyptera cell two sections of diverging axial core were visible in the chromatin (fig. 1(a)). In the late pachytene $M$. maculatus cell, no clear envelope attachment was visible at either end of the SC of the B chromosome, although both ends of the bivalent were adpressed to the nuclear envelope, and it may have been entering the diplotene state. The single terminal attachment, near-terminal centromere and short length of the SC of the B chromosome are all consistent with foldback or hairpin pairing.

\section{DISCUSSION}

The results reinforce the view that the Bs are isochromosomes (fig. 2(a)) and there is no evidence of differences in behaviour of the $B$ between the two species. The complete pairing at early zygotene (visible in E. brachyptera) occurs when pairing is expected to be restricted to homologous regions (Wettstein et al., 1984). The presence of two homologous arms within one chromosome helps to elucidate the mechanism by which chromosomes normally pair. The rapid pairing of B isochromosomes may be facilitated by having the centromeric ends of the arms held together (fig. 2(b)). However if pairing tends to start near the telomeres as is usual in grasshoppers (fig. 2(c)), the close association at the centromeric end may have little effect, and two B chromosomes could easily form a bivalent with telomeric pairing (fig. 2(d)). The B chromosome of $M$. maculatus is submetacentric. Hairpin pairing starting at the centromere would be unable to progress along the arms because of the extra material in the longer arm. If the inequality is due to a pericentric inversion, the long arm would contain a tandem duplication of sequences missing from the other arm (fig. 2(f)). This duplication would show an inversion loop if it paired homologously (fig. 2(g)) or a side hairpin loop if it failed to pair (fig. 2(h)). These structures were not found in the SC's reconstructed, and the centrometric ends of the SC's did not show an 

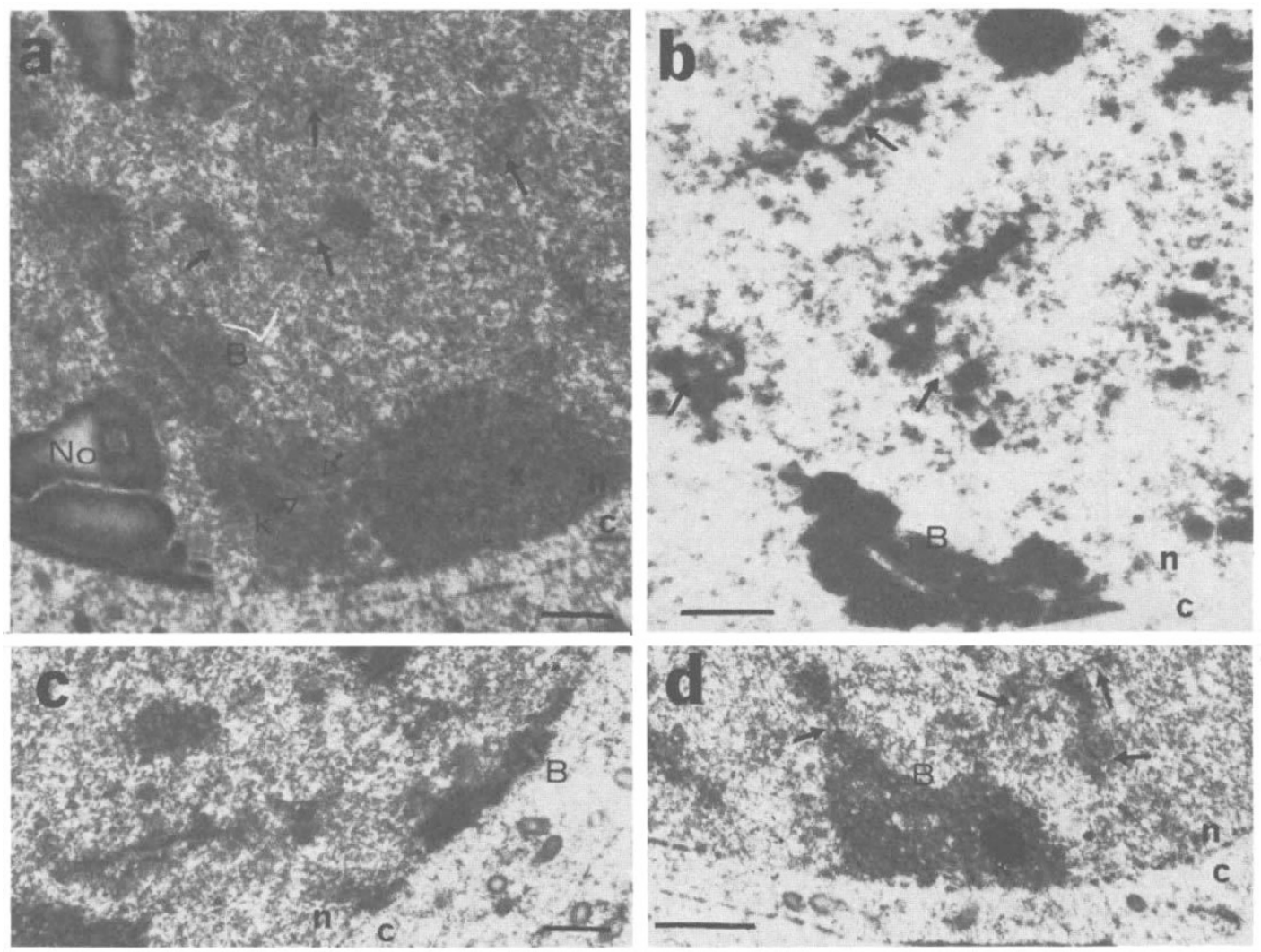

Figure 1 B Chromosomes (B) in meiosis in Euthystira brachyptera (a, c and d) and Myrmeleotettix maculatus (b). The greater degree of condensation of the B compared to the autosomes is evident in $a, b$ and $d$ where some of the autosomal SCs have been marked with arrows. The presumed kinetochores (k) of the Euthystira B univalents are shown in a and d. The B's unpaired axial cores can be seen diverging between the open arrows in a, indicating incomplete pairing. The distal end of the same SC is shown in $\mathrm{c}$ where it is attached to the nuclear envelope. No=nucleolus, $\mathrm{X}=\mathrm{X}$ univalent, $\mathrm{n}=$ nucleus, $\mathrm{c}=$ cytoplasm. Bars $=1 \mu \mathrm{m}$.

abrupt stop with the lateral element folded back consistent with pure isochromosome morphology (fig. 2(e)). Indeed in E. brachyptera cell 2 (fig. 1(a)) two unpaired axial cores can be seen diverging near the centromere consistent with a failure of pairing near the centromere (fig. 2(i)). This suggests that pairing is started near telomeres even in $\mathrm{B}$ isochromosomes.

In $M$. maculatus cells with 2B chromosomes, 40 per cent of meiotic cells contain a B-bivalent as opposed to univalent B's (John and Hewitt, 1965a) demonstrating that the isochromosome structure of B's in this species is not sufficient to force exclusive foldback pairing. The frequency of 2B cells with univalent B's is however greater than the one third expected from random pairing of arms, $\chi_{(1)}^{2}=17.8, \quad p<0.001 * * *$ suggesting that pairing is slightly more likely to occur between arms within a chromosome than between chromosomes.
The B chromosome in E. brachyptera has been observed in first and second divisions of meiosis, where there is no visible difference in the lengths of the arms. The single $2 \mathrm{~B}$ individual found contained seven observable meiotic cells and each contained two univalent B's.

It is commonly observed in grasshoppers that shorter chromosomes pair up first and rapidly acquire complete SCs (e.g., Fletcher, 1977; Jones and Croft, 1986). This holds true for E. brachyptera. The individual arms of the $B$ are similar in length to the S7 or M6 bivalent, and the rapid pairing of the Bs may be attributable to their small size rather than their morphology. In summary, it seems that meiotic pairing is not started where the homologous arms are held in close proximity at the centreomere, although, intrachromosomie pairing is preferred to interchromosome pairing.

Apart from the above conclusion that meiotic pairing is initiated away from the centromere in 

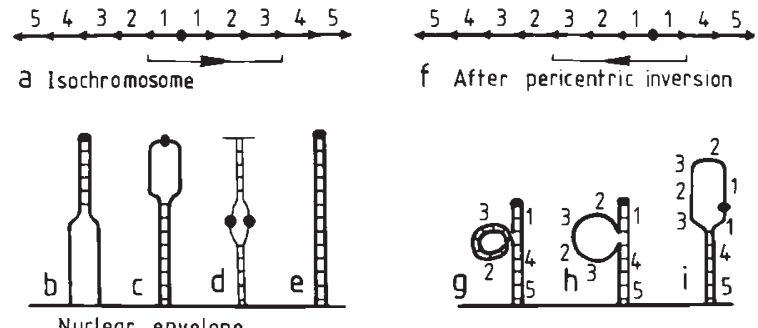

Figure 2 Possible pairing arrangements of isochromosomes. Centromeres are shown by solid circles and synapsed regions by cross bars. An isochromosome (a) has arms which are mirror images-inverted repeats. If homologous regions adjacent to the centromere pair then the structure in b will appear at zygotene, however if pairing starts at telomeres then the structure in c will result, and two B isochromosomes can form a bivalent (d). Complete pairing in an isochromosome would give a complete hairpin SC (e). M. maculatus B chromosomes are submetacentric, probably as a result of a pericentric inversion (f). The resultant duplication deletion could form a paired $(\mathrm{g})$ or unpaired (h) loop. Note that the relative sizes of numbered sections is arbitrary and section 1 in particular could be invisibly small. Reconstructed SCs were only consistent with an absence of pairing near the centromere (i) suggest ing only telomeric pairing.

B isochromosomes, evidence pertinent to two other phenomena of general importance is contained in these results. The first relates to chromosome condensation. The lengths of the SC's of particular bivalents varies both systematically between meiotic stages and also for no apparent reason between individual cells. This must be related to compaction of the lateral elements (chromosomal axes) but not enough is known about the structures involved to reveal the significance of the phenomenon. The precocious synapsis of the $B$ chromosomes is followed by equally precocious condensation which is visible by light or electron microscopy and is accompanied by an apparent shortening of their SC's relative to those of the autosomes. At early zygotene in E. brachyptera the $\mathrm{SC}$ of the B is 72 per cent of its relative mitotic length, and at late pachytene in $M$. maculatus it is about half of its expected relative length.

The second phenomenon is the distribution of chiastmata along bivalents, which is closely controlled. Shorter bivalents reguarly have one chiasma to facilitate disjunction, while multiple chiasmata on longer bivalents show positive interference; they are regularly spaced rather than randomly spaced. In some species (and sexes) chiasma localisation occurs; chiasmata are restricted to limited regions of the bivalent. For example, in the grasshopper Stethophyma grossum, males have terminal procentric localisation of chiasmata. Details of the mechanism controlling chiasma localisation are not known but in Stethophyma the SC is also terminally localised like the chiasmata (Fletcher, 1977; Wallace and Jones, 1978), the distal parts of the bivalents failing to pair. Male Euthystira are also noted for terminally localised chiasmata, particularly prominent in the M4 and M5 bivalents which usually have one chiasma at each end (Fletcher and Hewitt, 1980). However, pairing is completed early in zygotene in these bivalents (table 1 above) so localised crossing over is clearly not related to localised pairing in these bivalents, and the system operating here may be different from that in Stethophyma.

Acknowledgements This work was supported in part by an SERC grant.

\section{REFERENCES}

FLETCHER, H. L. 1977. Localised chiasmata due to partial pairing: A 3D reconstruction of synoptonemal complexes in male Stethophyma grossum. Chromosoma (Berl.), 65, 247-269.

FLETCHER, H. L. AND HEWITT, G. M. 1980. Effect of a "B" chromosome on chiasma localisation and frequency in male Euthystira brachyptera. Heredity, 44, 341-347.

JOHN, B. AND HEWITT, G. M. $1965 a$. The B-chromosome system of Myrmeleotettix maculatus (Thunb.) I. The mechanics. Chromosoma (Berl.), 16, 548-578.

JOHN, B. AND HEWITT, G. M. $1965 b$. The B-chromosome system of Myrmeleotettix maculatus (Thunb.) II. The statics. Chromosoma (Berl.), 17, 121-138.

JOHN, B. AND HEWITT, G. M. 1966 Karyotype stability and DNA variability in the Acrididae. Chromosoma (Berl.), 20 , 155-172.

JONES, G. H. AND CROFT, J. A. 1986. Surface spreading of synaptonemal complexes in locusts II. Zygotene pairing behaviour. Chromosoma (Berl), 93, 489-495.

JONES, R. N. AND REES, H. 1982 B Chromosomes. Academic Press, London.

WALLACE, B. M. N. AND JONES, G. H. 1978. Incomplete chromosome pairing and its relation to chiasma localisation in Stethophyma grossum spermatocytes. Heredity, 40, 385396.

WETtSTEIN, D. VON, RASMUSSEN, S. W. AND HOLM, P. B. 1984. The synaptonemal complex in genetic segregation. Ann. Rev. Genetics, 18, 331-414. 